\section{Medikamentöse ADHS-Behandlung senkt Kriminalitätsrate}

Etwa $5 \%$ aller Kinder in der westlichen Welt erfüllen die Kriterien für ein Aufmerksamkeitsdefizit-/Hyperaktivitäts-Syndrom (ADHS). Viele von ihnen werden mit Medikamenten behandelt. Sehr häufig wird die Behandlung jedoch in der Adoleszenz und im frühen Erwachsenenalter beendet. Das hat Folgen, wie eine schwedische Untersuchung zeigt.

ᄃine Arbeitsgruppe um Paul Liechten- stein vom Karolinska Institut in Stockholm ist der Frage nachgegangen, welche Auswirkungen eine Therapieunterbrechung bei ADHS auf die Kriminalitätsrate hat. Sie konnte dazu die Daten aus nationalen schwedischen Registern mit über 25.656 Patienten (davon 16.087 Männer) mit der Diagnose ADHS nutzen. Die Verbindung verschiedener $\mathrm{Da}-$ tenbanken erlaubte es, die Medikamentenbehandlung und Verurteilung wegen Straftaten miteinander zu verbinden. Dabei wurden Perioden ohne Medikation verglichen mit solchen mit Medikation.

Es zeigte sich, dass es bei Patienten unter Medikation zu einer signifikanten Verminderung der Kriminalitätsrate um $32 \%$ bei Männern und $41 \%$ bei Frauen kam im Vergleich zu Patienten, die die

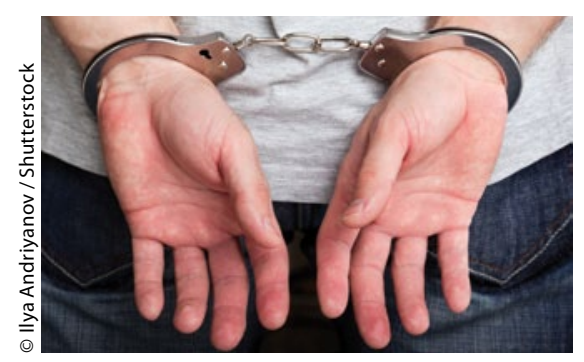

Wird die Medikation abgesetzt, steigt die Rate an Verbrechen.

Behandlung ausgesetzt hatten. Die medikamentöse Behandlung erfolgte vorwiegend mit Methylphenidat, seltener mit Amphetamin und Dextroamphetamin oder Atomoxetin. Der Endpunkt war die Verurteilung wegen einer Straftat. Jeder Patient diente als seine eigene Kontrolle. Die Verminderung der Kri- minalitätsrate war unabhängig davon, ob zur Therapie ein Stimulans oder Nicht-Stimulans genutzt wurde. Nachdem in Schweden eine Medikamentenbehandlung des ADHS nur durchgeführt wird, wenn zuvor andere Behandlungsmethoden versagt haben, ist davon auszugehen, dass in dem untersuchten Kollektiv die schwereren ADHS-Fälle enthalten waren.

Lichtenstein P et al. Medication for attention deficit-hyperactivity disorder and criminality. N Engl J Med 2012; 367: 2006-14

Kommentar: Es ist faszinierend, welche eindrucksvollen Erkenntnisse erzielt werden können, wenn genügend auswertbare Daten zur Verfügung stehen und unterschiedliche Datenquellen miteinander verknüpft werden. Die Autoren betonen allerdings auch selbst, dass die positiven Effekte der Medikamentenbehandlung sorgfältig abgewogen werden müssen gegenüber potenziellen Nachteilen. In diesem Zusammenhang weisen sie auf die Arbeit von Graham und Coghill hin [Adverse effects of pharmacotherapies for attention-deficit hyperactivity disorder: epidemiology, prevention and management. CNS Drugs 2008; 22: 213-37].

Dr. Hartmut Koch

\title{
Wie stehen Kinder mit ADHS zur Stimulanzientherapie?
}

In einer Publikation im British Medical Journal wurden die Ergebnisse einer Befragung von 151 Kindern mit ADHS aus England und den USA vorgestellt. Die Kinder sollten berichten, wie es ihnen unter einer Medikation geht.

$D$ ie von ADHS betroffenen Kinder im Alter von neun bis 14 Jahren gaben überwiegend an, dass sie sich mit Hilfe der Medikation in der Lage fühlen, sich zu bremsen und zu denken bevor sie reden. Sie hätten eine bessere Kontrolle über sich und könnten selbstbestimmt handeln - auch wenn sie beschließen, sich mit anderen Kindern zu schlagen. Sie leiden mehr unter dem Gerede über die Medikation als unter der Medikation selbst. Sie gaben weiterhin an, dass sie wenig über ihre Störung wissen und auch nicht wissen, warum sie behandelt würden. Sie würden gerne mitbestimmen, ob und wann sie die Medikation einnehmen müssten.

Kmietowicz Z. Children don't think that stimulant drugs for ADHD rob them of their "authenticity" and moral responsibility. BMJ 2012; 345: e6947

Kommentar: Es handelt sich um die erste umfangreiche Befragung von Kindern mit ADHS. Vor allem in der Laienliteratur wird gern geschrieben, wie die Autoren sich denken, dass sich die Kinder fühlen: als Roboter, nicht mehr "sie selbst", fremdgesteu- ert und emotional abgeflacht. Diese Vermutungen bestätigen die betroffenen Kinder in den nun veröffentlichten Interviews von llina Singh vom Londoner King's College nicht. Im Gegenteil: Sie haben den Eindruck einer besseren Eigenkontrolle. Das Ergebnis dieser Befragung beruhigt und bestätigt den klinischen Eindruck.

Zu denken gibt die Klage der Kinder, zu wenig in den therapeutischen Prozess mit einbezogen zu sein. Auch wenn es zeitaufwendiger ist, so sollte doch mehr darauf geachtet werden, das betroffene Kind mit in die Entscheidung einzubeziehen und eine gute Aufklärung über die Störung durchzuführen. Die Compliance dürfte sich dadurch sicher bessern. Das Ergebnis der Studie schließt nicht aus, dass es von ADHS betroffene Kinder gibt, die sich unter einer Medikation wie "Roboter" oder "Zombies" verhalten, dies dürfte aber die Folge einer Überdosierung von Methylphenidat sein.

Dr. Kirsten Stollhoff 